\title{
Face Recognition and Detection through Similarity Measurements
}

\author{
Irfan Bashir \\ M.Tech( CSE) Schoral \\ SMVDU, Kakryal Katra, Jummu
}

\author{
Ajay Koul, PhD \\ Director \& H.O.D of CSE Department \\ SMVDU, Kakryal Katra, Jummu
}

\begin{abstract}
The facial recognition has been a problem worked on around the world for many persons, the problem has emerged in multiple fields and sciences, especially in computer science and other fields that are very interested in this technology are robotic, criminalist etc. Unfortunately, many reported face recognition techniques relay on the size and representative of training set such as e-passport, law enforcement and id- card identification, and most of them will suffer serious performance drop if only one training sample per person is available to the systems [1].In a face image, only a part of face is changed due to pose, illumination and other source of changes .In this paper, a novel face recognition detection approach known as Gabor wavelet based PCA approach is presented based on fusing global and local features of image. To extract global and local features, Gabor wavelet filter are applied on the whole image and non-overlapping sub- images with equal size. To reduce the dimension of new fused feature vector and to better characterize the similarity between each gallery face and the probe image set, Principal Component Analysis (PCA) is employed. And finally, measure the similarity between the images by using the Euclidean distance as classifier. The Experimental results shows that proposed technique improves the efficiency of face recognition under varying illumination, expression and variation in poses of face images by using standard databases when compared to traditional PCA and Conventional method such as global Gabor faces recognition. In this paper, the proposed algorithm is tested on the public and largely used ORL database.
\end{abstract}

\section{Keywords}

Face recognition, Face detection, PCA, Eigenfaces, Gabor Wavelet, Gabor faces, Dimensionality reduction.

\section{INTRODUCTION}

Facial recognition technology attempts to match faces to determine if the person shown in one image is the same shown in another image. It is actually, a real-time operation for automatically identifying or verifying a person from a digital image or a video frame from digital cameras. This is distinct from face detection which only determines where in an image faces are present. Face detection is an important part of face recognition or is first phase of automatic face recognition (in subsec.2.4.2 and 3.1.1, some discussion about it is written). However, face detection is not straightforward because it suffers lots of variations of image appearance, such as occlusion, orientation, illumination and facial expressions. Automatic face recognition is one of the fundamental problems in computer field and pattern analysis from over decades. During past 20 years, this technology have received significantly increased attention from both the industrial and academic communities with emphasis on such applications as biometric analysis, context based coding of images and videos, human computer interaction and surveillance. Developments in this field have been categorized into modes-
Appearance based and Model based Algorithms, Gabor wavelet based PCA approach follows the former mode.

Face recognition problem can be defined as follows: Given an input face image and a database of face images of known persons, to verify or to determine the identity of the person in the input image? Nowadays, due to the security issues such as, problem caused by stolen and loss of passwords in many domains, research efforts have been focused on how to improve the accuracy of a recognition and detection system to identify a person from the database later in time in any different and unpredictable poses, expressions and lighting etc. In recent years, many conventional techniques have been developed to attack this problem, such as Holistic and feature based [2-4], probabilistic matching [5,6], and neural network methods $[7,8]$.

This paper, presents a new face recognition and detection algorithm based on global and local features as Gabor wavelet based PCA approach. Due to changes in only some region of face, the local features are used. Initially, segment the face images into non-overlapping smaller sub-images called modules. Each of these modules is represented by Gabor wavelets $[9,10]$, an effective approach for feature extraction and then PCA technique is applied for dimensionality reduction. Finally, in recognition phase, classification is done based on a Minimum distance Classifier [11].In this paper, a Standard database-ORL database (that holds detection phase) are used for experimental results.

\subsection{Related Work}

Face Recognition and Detection has been a significant problem in Image processing and pattern recognition over the last several decades. It plays an important role in many applications such as security monitoring card identification, access control and mug shot searching etc. Face recognition and detection system is considered as one of the most important system which can be affected by variations [12][14].However to obtain best recognition and detection performances, many achievements has been made by researchers.

Manjunath et al. [15] proposed a method for facial feature detection and representation, based on Gabor wavelet decomposition of the face. For each detected feature points, two types of information are stored, i.e., location information and feature information and a topological graph is constructed to model the relationship among the feature points by connecting two feature points in some spatial range with minimal distance with an edge. After that, face recognition is formulated as a graph matching problem. [16] describes a multi-model method for personal identification and verification, using at least two biometric readings: First, a biometric reading(e.g. iris scan) is processed to create the non-reversible ID from a biometric samples (Enrollment module).The second (verification-module) performs the 
identification process. It was resulted that the method does not rely on the network infrastructure or on the databases to perform authentication or verification.

Davida et al. [17] proposed that process of generating cryptographic keys from biometrics generally relies on an error tolerant binary representation of the biometrics features by employing hash functions to cope with both problems: variability of biometric templates and protection of personal data. Furthermore, error correction codes are employed in order to extract an unique associated feature from each different biometric reading: the different readings are treated as corrupted code words and are accordingly decoded. During the verification phase, the feature retrieved by a biometric reading is given as input to a hash function, and compared with the hash value stored during the enrollment phase.

In [18] recent trends to automatic recognition of human facial behavior (internal mood or feelings) using Soft computing techniques are described. Soft computing techniques are constituted of fuzzy logic, neural network and genetic algorithms are applicable in the problem whose solution is uncertain (approximation).

[19] This paper presents a fast and accurate method for tracking (detecting) and recognition of human motion from a video stream or in any environment i.e. indoor/outdoor scenes and occluded regions. A motion tracking system is developed to determine the trajectories of each player whether occlude or overlapped from image sequences of sport games video footage. In this method, Singular value decomposition of Gaussian Elimination method is employed to find the selected person from the two images.

Sakai et al. [20] introduces the edge map extraction from an input image and then matching it to a large template, with possible variations in position and size. Kelly [21] introduced an improved edge detector, involving heuristic planning to extract an accurate outline of a person's head from various backgrounds.[22] addresses the very low resolution problems in face recognition in which the face image resolution is lower than $16 \times 16$ by a novel approach-SR algorithm based on relationship between high resolution image space and VLR image space.

The rest of paper is organized as follows: a general overview of the face recognition is presented in section 2 , Section 3 consists of the proposed algorithm. The image databases used in the experiments are presented in section 3.1.2. Results and the statistical analysis is given in section 5 followed by conclusion in section 6 .

\subsection{Why Similarity Measures are Useful?}

Nowadays, there are various computer imaging application that requires some kind of similarity measurement comparison as a part of their processes such as comparison of frames in a video, pattern recognition approach, image stabilization using a homographic transformation and using image feature points to compute similarities to generate an image mosaic. Although, the Applications are quite varied and their implementation processes of each solution are unique but all shares the common thread in that features or attributes in which the image are measured and then compared to the other features from a database of images or reference model to extract meaningful information about the image data.

Specially, when designing a system that requires image comparison, one main objective is to find a feature calculation that will help accurately to measure image similarity. The second property of feature computation, especially when dealing with video or the large database is selecting a feature such that the data size of feature is smaller than the original data. For instance, in image matching application based on sample query images, it is efficient to compare features extracted from sample image with features extracted from each image in the database. This method is totally opposed to comparing the entire sample image to each original image in the database, since the features that describes the image is smaller in magnitude than the sampler image.

In addition to compute homographs between images such as, in image search on internet and in arty application such as, to generating image mosaics (reconstructing a still target image from a database of tiled images or thumbnail)[23][24] image similarity metric are used to compute similarities in images. Thus, using similarity measurements are typically found more efficient in image processing applications that require image similarity comparison or intermediate feature space as opposed to comparing the image pixels directly.

\section{FACE RECOGNITION AND DETECTION -A GENERAL VIEW}

\subsection{Why to Select Face for Recognition?}

In recent years, increased demand of security in various domains made researchers attractive towards developing the technologies that can recognize or authenticate people or individuals, and granting them access to physical and virtual domain based on PINs, Keys, retina ,gait, face, signature and so forth. Biometric-based technologies, ascertain identification of individuals based on physiological characteristics (such as, finger and hand geometry, palm, face, fingerprints, iris, retina and voice etc.) and behavioral traits (such as, signature, gait and keystrokes etc.)[25] but almost requires some voluntary actions by the individuals i.e. the user need to stand in a fixed position in front of camera for iris or retina verification or has to place his hand on hand-rest for fingerprinting and hand geometry detection. Opposed to it, face recognition can work passively by obtaining facial images with couple of inexpensive cameras. Biometric techniques: one that rely on hand/finger can rendered useless data if epidermis is damaged (due cracking), signature be modified or forged, and the others that use same equipment to capture biological characteristics of multiple individuals can potentially expose the individual to the transmission of germs and infection from other individual. However, face recognition technique can compensate noise and slight variations in scale, illumination or expression etc., thus are non-intrusive without any health hazards.

\subsection{General Problems in Face Recognition.}

Although most face recognition techniques work well under constrained conditions, the performance of most these techniques degrades rapidly if none these factors are regulated[26].The difficulty of this technology stems from the fact that the human face is not unique, rigid object. Truly, there are various sources of variation that causes the facial appearance to change, can be categorized into two groups: a) Intrinsic and b)Extrinsic factors[27].Intrinsic factors include physical nature of face and are independent of observer while as extrinsic factors causes the alternation of face through the interaction of light with face and the observer. Intrinsic factors are further categorized into subgroups [28]:i) Intrapersonal factors responsible for varying of facial appearance of same person and ii)Interpersonal factors defines variation of facial 
appearance between different persons. Moreover, the performance review of FERET, FRVT2002 [29] and FAT 2004 databases have confirmed that illumination, pose(expression) and age variations are the three main serious problems affecting the current face recognition systems[30].

\subsection{Structure Process of Face Recognition System.}

In modern times, demand for enhanced security in public interest, face recognitions system has become particularly important. It can operate in either or both of two modes:

i) Face verification (or authentication): involves a one-to-one match that compares a query face image against a template face image whose identity is being claimed.

ii) Face identification (or recognition): involves oneto-many matches that compare a query face image against all the template images in the database to determine the identity of the query face.

First automatic face recognition system was developed by Kanade 1973.

Face recognition system can broadly be classified as consists of two main phases namely, Training phase and Testing phase with at least four modules as:

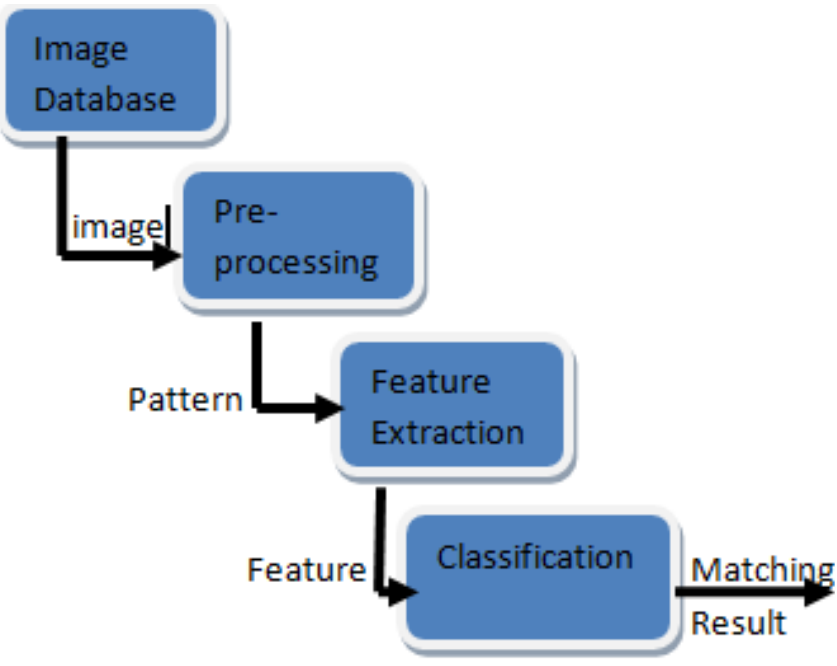

Figure1. Structural process of face recognition system.

\section{i) Image database:}

This stage is simple because it only needs a digital input device to take the face image to be processed. Surely, if the camera has a better resolution clearer images can be obtained for processing.

\section{ii) Preprocessing:}

This stage is performed to obtaining the specified result images. Noise removing, thresholding and binarilzation,
Grayscale converting, histogram equalization, edge detection, smoothing and cropping are done for convenient extraction of feature from any input image.

\section{iii) Feature extraction:}

It is one of the most important stages in the recognition systems because at this stage facial features are extracted in correct magnitude or dimension to give a good representation of the characteristic information of the person that will serve to have a good training of the classification models. Today, great diversity of feature extraction algorithms, are listed some of them as:

- Fisher faces (Alvarado et al., 2006).

-Eigenfaces (Alvarado et al., 2006).

- Gabor Filters (Olivares et al.2003).

-Discrete Walsh Transform (Yoshida etal.,2007).

- Discrete Wavelet Transform (Bai-Ling et al., 2004).

\section{iv)Classifier:}

A classifier is an algorithm to define a model for each class (object specific), and its goal is to assign a name to a set of data for a particular object or entity so that the class to which it belongs an element can be calculated from the data values that define the object.Usually, also considered a test set that allows to validate and to measure the accuracy of the model. Presently, supervised and unsupervised are different ways of learning for classifier. The following are the different types of classifiers exist:

- Nearest Neighbor (Davies E. R., 1997; Vladimir and

Filip, 1998)

-Bayes' Decision (Duda et al., 2001).

-Neural Network (Rojas R., 1996).

-Support Vector Machine (Vladimir and Filip, 1998).

-Gaussian Mixture Models (Reynolds D. A., 2008).

\subsection{Face Recognition and Detection techniques.}

2.4.1 Face Recognition Techniques:-

Depending on the methods for acquisition of face data, face recognition techniques can broadly be categorized into three categories:

A) methods that operate on intensity images,

B) those that deal with video sequences, and

C) the methods that require other sensory data such as $3 \mathrm{D}$ information or infra-red imagery. Face recognition methods for intensity images are divided into two categories:

a) feature based and

b) holistic [2-4], whereas holistic approaches are sub-divided into two groups-Statistical and AI approaches. The following tabular representation shed some light on the methods in each category as follows:

\section{Approach Representative works}

Feature methods:

Feature based methods

Elastic bunch graph matching method
Kanade[31]

Wiskottetal[32,33] 
Feature graph based

Fractal features based methods

Holistic methods: a) Statistical approaches:

PCA

Independent component analysis (ICA)

Linear discriminative analysis (LDA)

Gabor-Fisher, ICA

Gabor-Kernel PCA

Gabor-GDA

\section{b) AI methods:}

Hierarchical neural network

Hybrid neural network

PCA using feed forward neural network

Local binary pattern histogram

Adaboost learning algorithm

Hidden morkov models

GWN

[51]

SDGWN + KAM

Face recognition from video sequences:

Two layer RBF network

Skin color modeling and GWN

A detailed survey of schemes for face recognition from video stream is provided in [55].

Face recognition from other sensory inputs:

3D model-based or range data

Principal curvature of face surface

3D face recognition based on local features

[59]

Comprehensive survey on 3D face recognition can be found in $[60,61]$

Face recognition from infra-red imagery:

Eigen face techniques $[62,63]$

Socolinsky et al. [64] [65]

\subsubsection{Face Detection Techniques:-}

The Face detection techniques can broadly fall into following categories:

i) Appearance-based and learning based approaches.

ii) Preprocessing.

iii) Neural networks and kernel-based methods.

iv) AdaBoost- based methods.

v) Dealing with head rotations

vi) Performance evaluation.

Most successful face detection algorithms are appearance-based without using any cues e.g. skin color, motion facial/head shape etc. Some approaches are as:

-Principal component analysis (PCA) or eigenfaces representation method [66, 67].

- Bayesian Discriminating Features (BDF) approach [68, 69].

- Boosting algorithm, called Float Boost approach [70].

\section{PROPOSED METHOD-THE GABOR WAVELET BASED PCA APPROACH}

\subsection{Structural Process of Proposed Method.}

The process of training and recognition (testing) phases of our proposed technique is as shown: 


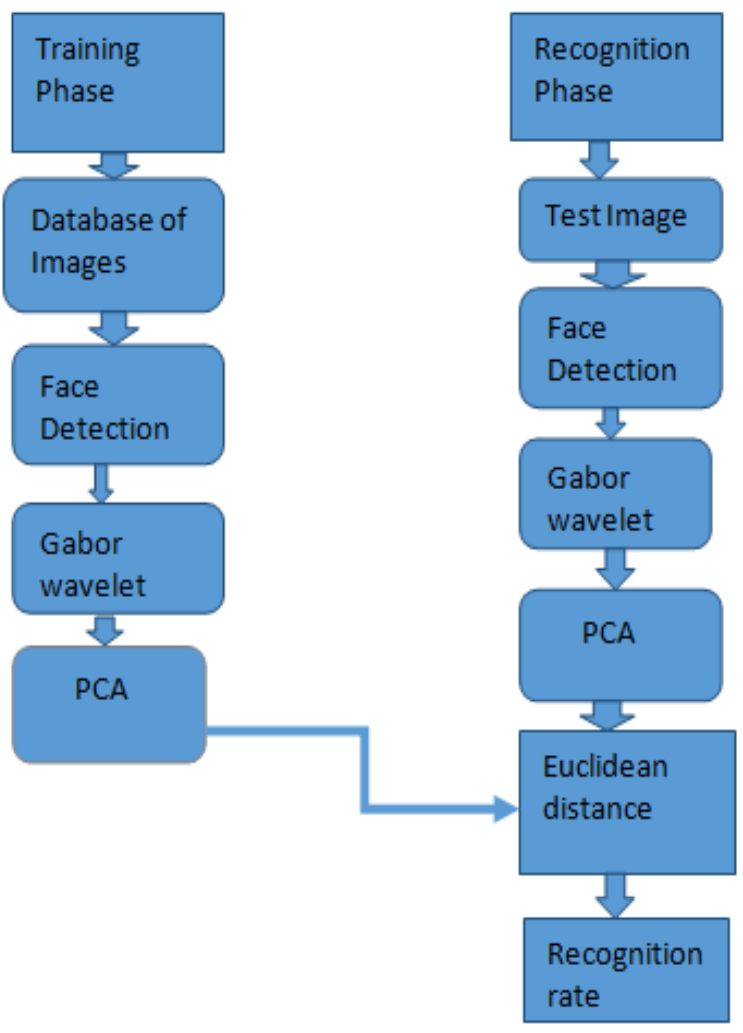

Figure 2.Structural process diagram.

\subsubsection{Face Detection:}

Face detection is the first step in automated face recognition. The purpose of face detection is to find whether there exists any faces in an image and if any, show location. Face detection can be performed based on several cues: Skin color, motion, facial/head shape, facial appearance or a combination of these parameters. In detection process, an input image is scanned at all possible locations and scales by a sub-window. Face detection is posed as classifying the pattern in the subwindow as either face or non-face.

Large variations brought about by changes in facial appearance, lighting and expression make the face manifold or face/non-face boundaries highly complex. Open $\mathrm{CV}$ is an open source computer vision library written in $\mathrm{C}$ and $\mathrm{C}++$ and runs under Linux, Windows and Mac OS X [71].Paul Viola initially proposed object detector of Open $\mathrm{CV}$ and later improved by Rainer Lienhart [72].

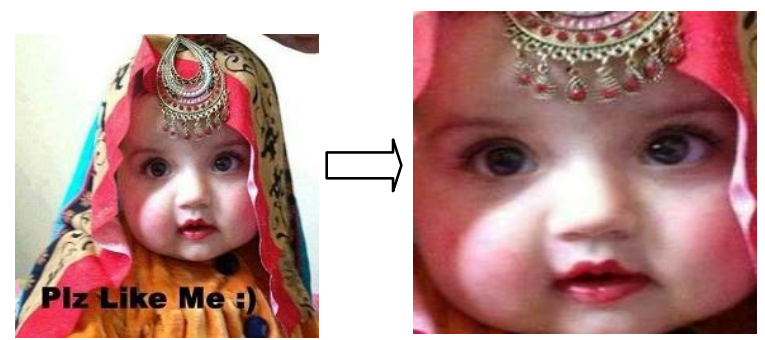

Figure 3. Detection face with open cv.

\subsubsection{ORL Database:}

To evaluate the performance of proposed methodologyGabor Wavelet based PCA approach we perform the test on ORL face database which contains 400 images of 40 subjects, with 10 different images of each subject. These face images of each subject were taken at different times, varying the lighting, facial expressions (smiling / not smiling, open/ closed eyes,) and facial details (glasses, no glasses)[73].

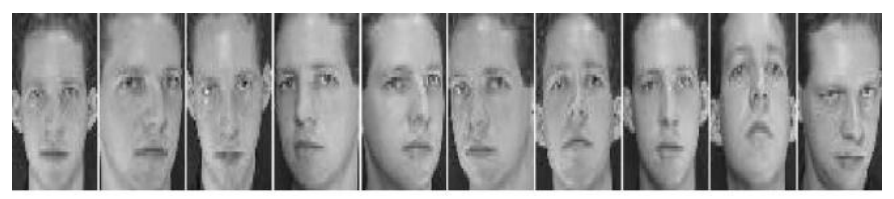

Figure 4. Some samples of one subject in ORL Database.

\subsection{Principal Component Analysis:}

Principal Component Analysis (PCA) was invented in 1901 by Karl Pearson. It is the simplest, mostly true eigenvectorbased multivariate tool in exploratory data analysis and for making predictive models (e.g., Face recognition). Usually, its operation can be thought of as revealing the internal structure of data in such a way that best explains the variances (major features/directions) in the data.

\subsubsection{An Overview of Principal Component Analysis:}

When a multi-variant dataset or training set (consists of set of images) is viewed as set of coordinates in a high dimensional data space(1- axis per variable), PCA transforms this training set of images into a lower dimensional picture or features, a "shadow" of this object when viewed from its(in some sense), a most informative view point. This transformation is defined in such a way that the First Principal Component (lower dimensional picture or eigenfaces) show the most dominant " features/directions" of the data set and each successive principal components in turn shows the next most possible dominant "features", under the constraint that it be uncorrelated the preceding components as in figure 5.As, each preceding principal components shows less" features/directions" and more "noise", only few first principal components(say K) are selected where as the rest of the last components are discarded as shown in figure 6.These ' $\mathrm{K}$ principal components can safely represent the whole original data set as, they depicts the major "features/ directions" that makeup data set.

This makes the recognition process faster and more free of 'errors' caused by noises.

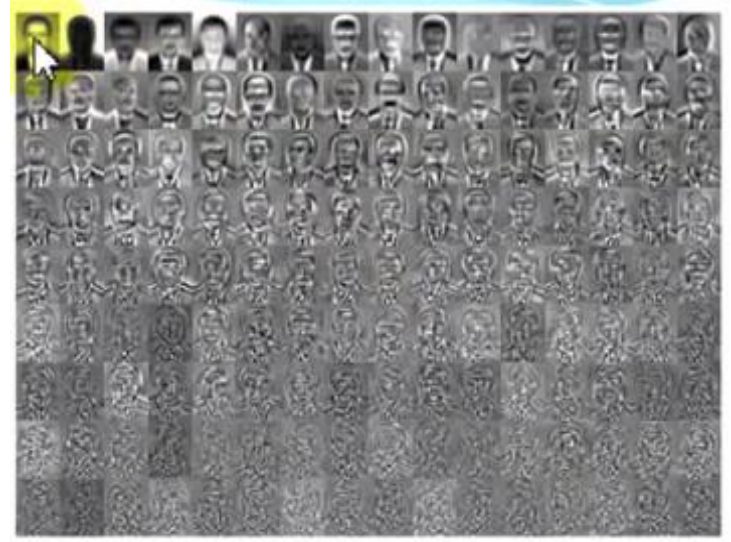

Figure 5. First Principal Component show the most dominant features 


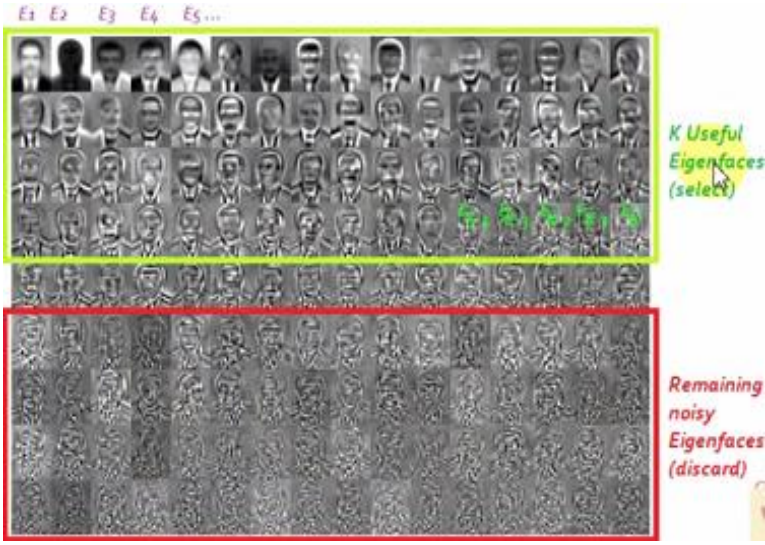

Figure 6. Selecting of Eigenfaces

\subsubsection{Mathematical Analysis of Principal}

Component Analysis:

The Principal Component Analysis (PCA)can be thought as a mathematical procedure that uses an orthogonal transformation to convert a set of possibly correlated variables(face images) into a set of values of uncorrelated variables (lower dimensions or eigenfaces), also called Principal Components. The number of principal components/eigenfaces are always less or equal to the number of original variables i.e., ' $\mathrm{K}$ ' selected (Eigen faces) are less than or equal $\mathrm{M}$ (original faces). The aim of using the PCA for face recognition is actually to express the large 1-D vector of pixels from 2-Dfacial image into the compact principal component of feature spaces, named as eigen space projection. Eigen space is calculated by finding the eigenvectors of the covariance matrix of facial images. Usually, it is difficult to depict a suitable threshold [74] for recognition.

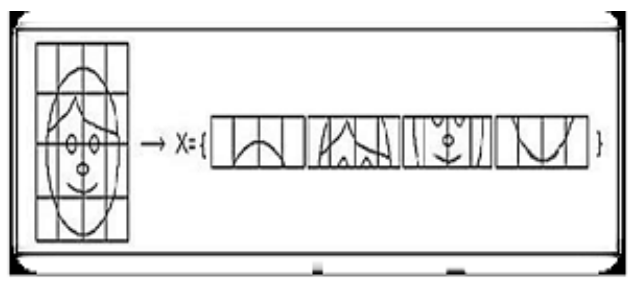

Figure 7. Face image viewed as vector.

Let $\mathrm{I}=(\mathrm{x}, \mathrm{y})$ be a two-dimensional (2-d) face image matrix of size $\mathrm{m} * \mathrm{n}$ and $\mathrm{I}_{1}, \mathrm{I}_{2}, \mathrm{I}_{3} \ldots .$. In denote $\mathrm{N}$ face images. Then Ii can be represented by a one dimensional (1-d) matrix:

$$
\mathrm{Ii}=\left[\begin{array}{c}
\mathrm{I}_{1} \\
\mathrm{I}_{2} \\
\mathrm{I}_{3} \\
\cdot \\
\cdot \\
\cdot \\
\mathrm{Imn}
\end{array}\right]
$$

And the face image-set is given by:

$$
\mathrm{I}_{\mathrm{i}}=\left[\mathrm{I}_{1}, \mathrm{I}_{2}, \mathrm{I}_{3}, \ldots . . \mathrm{In}\right]
$$

\section{Step 1: Get some data:}

A self-made data set consists of $\mathrm{N}$ images (have 2-D only) is used to show the PCA analysis at each step. This training set is then converted into vector form, as PCA does not work directly on images.

\section{Step 2: Normalize:}

Normalize the face vectors by calculating Average of all training samples (Im):

$$
\operatorname{Im}=\frac{1}{\mathrm{~N}} \sum_{\mathrm{i}=1}^{\mathrm{N}} \mathrm{I}_{\mathrm{i}}
$$

\section{Step 3: Subtract the mean:}

Then, subtract the mean(average) from each face vector to get the normalized training sample:

$$
\emptyset_{\mathrm{i}}=\mathrm{I}_{\mathrm{i}}-\mathrm{I}_{\mathrm{m}}
$$

Where the column vectors $\emptyset_{\mathrm{i}}$ represents training sample and $\mathrm{Ii}^{\text {th }}$ face vector.

Step 4: Calculate the Eigen vectors:

To calculate the Eigen vectors, we need to calculate the Covariance matrix $\mathrm{C}$.

$$
\mathrm{C}=\mathrm{AA}^{\prime}
$$

Where $\mathrm{A}\left[\varnothing_{1}, \emptyset_{2}, \emptyset_{3} \ldots . \varnothing_{\mathrm{n}}\right]$ is nothing but consists of columns of normalized face vectors of images and of size $\mathrm{B} * \mathrm{~N}$, and the dimension of $\mathrm{C}$ is $\mathrm{B} * \mathrm{~B}$.

\section{Step 5: Select the Significant eigenfaces:}

As principle of PCA face recognition signifies to represent each image in a training set as linear combination of $\mathrm{K}$ selected eigenfaces, where $\mathrm{K}<\mathrm{n}$ we need find the $\mathrm{K}$ selected Eigen values (significant eigenfaces). The solution would be dimensionality reduction, this dimensionality reduction is done by simply calculating the Eigen vectors from a Covariance with reduced dimensionality. This Covariance matrix is of different formula:

$$
\text { i.e. } \mathrm{C}=\mathrm{A}^{\prime} \mathrm{A}
$$

$\mathrm{A}^{\prime}$ is of dimension $\mathrm{N} * \mathrm{~B}$, and the dimension of this covariance matrix is $\mathrm{N} * \mathrm{~N}$ will generate eigenfaces lesser than Covariance matrix $(\mathrm{B} * \mathrm{~B})$ and also reduces the size of eigen space.

Note: To reduce the calculation and affect of noise on the needed eigen vectors, calculate then from a Covariance matrix of reduced dimensionality.

\section{Step 6: Convert lower dimensional K eigen vectors to original face dimensionality:}

After finding the eigen values (significant), map back the eigen values to original dimensionality and is done, as we know,

$$
\mu_{\mathrm{i}}=\mathrm{A} \vartheta_{\mathrm{i}}
$$

Note: Eigen faces must be in original dimensionality of face vector space.

Where $\mu_{\mathrm{i}}$ and $\vartheta \mathrm{i}$ depicts the eigen vector in higher and lower dimensional space, that save the lot of computation and reduces the effective noise. So, has dimensionality reduction helped.

Step 7: Represent each image a linear combination of all $K$ eigen vectors:

After selecting $\mathrm{K}$ eigenfaces, each face from training set can be represented a weighted sum of the $\mathrm{K}$ eigenfaces plus the mean face (average).For this, add back the average faces that 
are subtracted in step 3(because we need add back the features that are removed before calculation). To do this, $\mathrm{K}$ weights (proportions of eigenfaces) are assigned to $\mathrm{K}$ eigenfaces.

$$
\text { Weighted vector } \Omega_{\mathrm{i}}=\left[\begin{array}{c}
\mathrm{W}_{1} \\
\mathrm{~W}_{2} \\
\mathrm{~W}_{3} \\
\cdot \\
\cdot \\
\mathrm{W}_{\mathrm{k}}
\end{array}\right]
$$

A weighted vector $\Omega \mathrm{i}$ which is the eigenface representation of the $\mathrm{i}^{\text {th }}$ face weight vector for each face is calculated. This is what the PCA results i.e. it finally represents each image of training set in form of Eigen- vector faces.

\subsection{Gabor Wavelet}

\subsubsection{Overview of Gabor Wavelet:}

The Gabor Wavelet technique instead of being used for face recognition, tracking and position estimation of faces, also have its wonderful characteristics. The use of 2D Gabor Wavelet representation of images in computer vision was pioneered by Daugman in 1980s[75].Gabor wavelets(also called Filters) have also been found appropriate for texture representation[76] and discrimination and for finger print recognition. Gabor filters exhibit desirable characteristics of Spatial frequency(scaling),localization and Orientation selectivity and Gabor filter representation of face images(Gabor faces) are robust for illumination and expressional variability, so are used for face recognition. The dimensionality of Gabor feature space are intensively high, because Gabor faces are obtained by convolution of the face with the dozens of Gabor wavelets(filters).So ,compressed methods are employed to reduce the space dimension to avoid dealing with enormous data $[77,78,79]$. PCA, 2DPCA, LDA are most popular used dimension reduction algorithms [77, 78,79]. And, we applied PCA algorithm toper form dimension reduction for feature extraction.

We adopt 40 Gabor Wavelets corresponding to 5 Scales and 8 orientations to convolve sample images because selection of Gabor 5 scale and 8orientations is the common characteristic in most existing face recognition systems. To improve the recognition performance, other feature extracted (or dimensional reduction) methods also can be used Such as, EDRS (Ensemble Dimension Reduction Scheme), MDRA (Multichannel Dimension Reduction Scheme). However, it is not the focus of this paper. Figure below shows the global and corresponding local sub -image Gabor features of whole image:

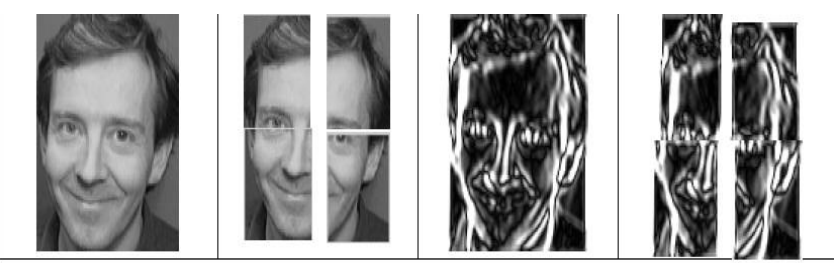

a) Test image b) Sub-images c)Global Gabor image d) Local Gabor Image

Figure 8.Global and corresponding Local sub-image Gabor Feature.

\subsubsection{Gabor Feature Extraction:}

Gabor Wavelet is a plane wavelet filter based on Gaussian kernel envelop modulated by a sinusoidal plane wave. In spatial domain, the commonly used Gabor kernel is defined as follows:

$$
\begin{aligned}
& \psi_{u, v}(Z)= \\
& \left(\frac{\|K u, v\|^{2}}{\sigma^{2}}\right)\left(\frac{\exp -\|K u, v\|^{2}\|x\|^{2}}{2 \sigma^{2}}\right)\left\langle\exp \left(i K_{u, v} . X\right)-\exp \left(-\frac{\sigma^{2}}{2}\right)\right\rangle \\
& \ldots \ldots . . . \text { Eqt. (I) }
\end{aligned}
$$

Where $\mathrm{Z}=(\mathrm{x}, \mathrm{y})$ is the point with horizontal and vertical coordinate $\mathrm{x}$ and $\mathrm{y}$, dot denotes norm operator, $\mathrm{u}, \mathrm{v}$ are orientation and scale of the Gabor filter, $\mathrm{Ku}, \mathrm{v}$ (wave vector) is defined as:

$$
\mathrm{Ku}, \mathrm{v}=\mathrm{K}_{\mathrm{V}} \exp \left(\mathrm{i} \emptyset_{\mathrm{u}}\right)
$$

Where, $\mathrm{K}_{\mathrm{V}}=\mathrm{Kmax} / \mathrm{f}_{\mathrm{v}}$ and $\quad \emptyset \mathrm{u}=\pi / 8,\|\mathrm{Ku}, \mathrm{v}\|^{2}$ ensures that filters tuned to different spatial frequency band (equal energy approximately), Kmax is the max frequency, and ' $\mathrm{f}$ ' is the spacing factor between kernels frequency domain. And $\sigma$ is the standard deviation of Guassian, thus constitute the kernel DC free. Therefore, it is unnecessary to consider the DC effect.

The first term in Equation (I), exp(iKu,v.x) controls the oscillatory part of the kernel and the second term $\exp (-\sigma 2 / 2)$ is subtracted, so that filters are intensive to the overall level of illumination i.e. compensate for the DC value. In most applications, parameters are set as follows:

$$
\sigma=2 \pi, K_{\max }=\pi / 2, f=\sqrt{2}
$$

The most extensively used selection of Gabor scale frequency and orientation is to select 5 scales and 8 orientation i.e. $v \in$ $(0,4)$ and $u \in(0,7)$.To represent Gabor Wavelet of an image, it is enough to convolve theimage with the family of Gabor Wavelets (kernels), defined in equation (I).The convolution of image and a Gabor kernel $\psi_{\mathrm{u}, \mathrm{v}}$ is defined as:

$$
\mathrm{I}_{\mathrm{u}, \mathrm{v}}(\mathrm{Z})=\mathrm{I}(\mathrm{Z}) * \psi_{\mathrm{u}, \mathrm{v}}(\mathrm{Z})
$$

Where, $\operatorname{Iu}, \mathrm{v}(\mathrm{Z})$ represents the result of convolution of the Gabor filter with orientation $\mathrm{u}$ and scale $\mathrm{v}, \mathrm{u} \in=(0,1, \ldots ., 7)$ and $\mathrm{v} \in=(0,1, \ldots ., 4)$.therefore, the set say,

$S=\{\operatorname{Iu}, v(Z): u \in(0,7), v \in(0,4)\}$ forms the Gabor Wavelet representation of the image $\mathrm{I}(\mathrm{Z})$, *a convolution operator.

In order that, this Gabor representation encompasses the information of different spatial frequencies (scales),spatial localities and orientations selectivity, a discriminative feature vector $\mathrm{I}^{\mathrm{r}}$ is derived to obtain an image ' I' by concatenating the vectors $\mathrm{Iu}, \mathrm{v}$, which are convolution result ,

$$
\left.\mathrm{I}^{\mathrm{r}}=\left(\left(\mathrm{I}_{0}, 0\right)^{\mathrm{T}} \quad\left(\mathrm{I}_{0}, 1\right)^{\mathrm{T}}\right) \ldots \ldots \ldots\left(\mathrm{I}_{4,7}\right)^{\mathrm{T}}\right)
$$

Remark: Therefore, by using 5 scale and 8 orientation, we obtain 40 Gabor features extracted for each of the sub-image that is convolved with the Gabor Wavelets (filters), so as vectors of long length are generated. After it, we apply PCA on these long vectors for dimensionality reduction. 


\section{A SUMMARIZE OVERVIEW OF PROPOSED APPROACH-GABOR WAVELET BASED PCA APPROACH}

The proposed approach can be summarized into five steps as follows:

I. First, the Global Gabor features are extracted by applying Gabor Wavelet filter on whole image of size say $m * n$. As a result, the global feature matrix $\mathrm{G}$ is extracted.

II. Local Gabor features are extracted by applying Gabor Wavelet on each sub-block of image, and get local Gabor matrices $\mathrm{G}_{1}, \mathrm{G}_{2}, \mathrm{G}_{3}, \mathrm{G}_{4}$.

III. In this step, the global and local features obtained from above two steps are combined to form a new feature vector

$$
\mathrm{I}=\left(\mathrm{G}_{1}, \mathrm{G}_{2}, \mathrm{G}_{3}, \mathrm{G}_{4}\right)^{\mathrm{T}} \text {. }
$$

\section{Dimension reduction:}

The new feature vector calculated in step-III has a high dimension. So, to increase the rate of recognition and to reduce the probability of over fitting, dimension of fused vector is reduced by using dimension reduction methods and we used PCA dimension reduction in our work.

$V$. And at this phase, comparison of fused feature vector of test image with fused feature vectors of data located in training database are performed.

\section{EXPERIMENTAL RESULTS}

This paper presents some computational results in Mat lab when we have tested the performance rate of our proposed method (Gabor Wavelet based PCA Approach) as shown:

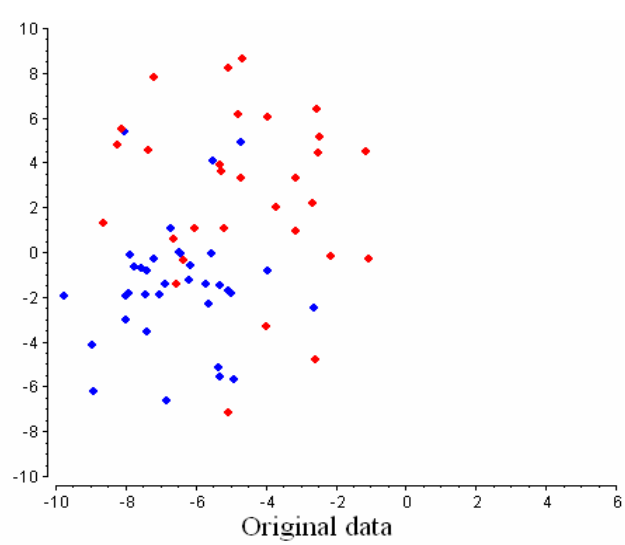

Figure 9(a)

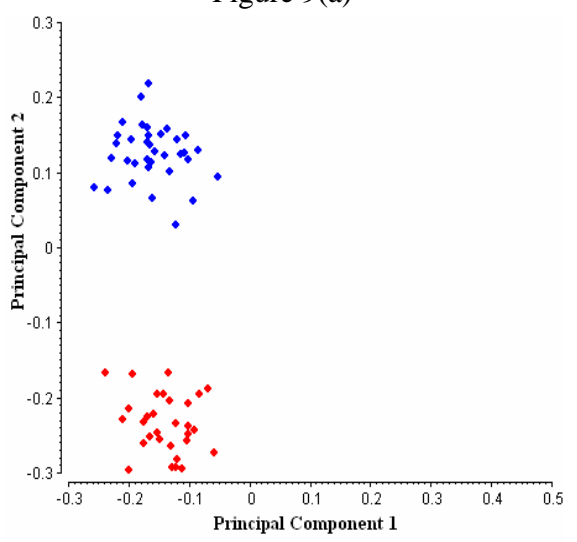

Figure 9(b)

Figure 9.An Example of PCA Analysis:

Figure 10.Magnitude part of representation and Phase part of representation for Gabor Wavelet shown as:

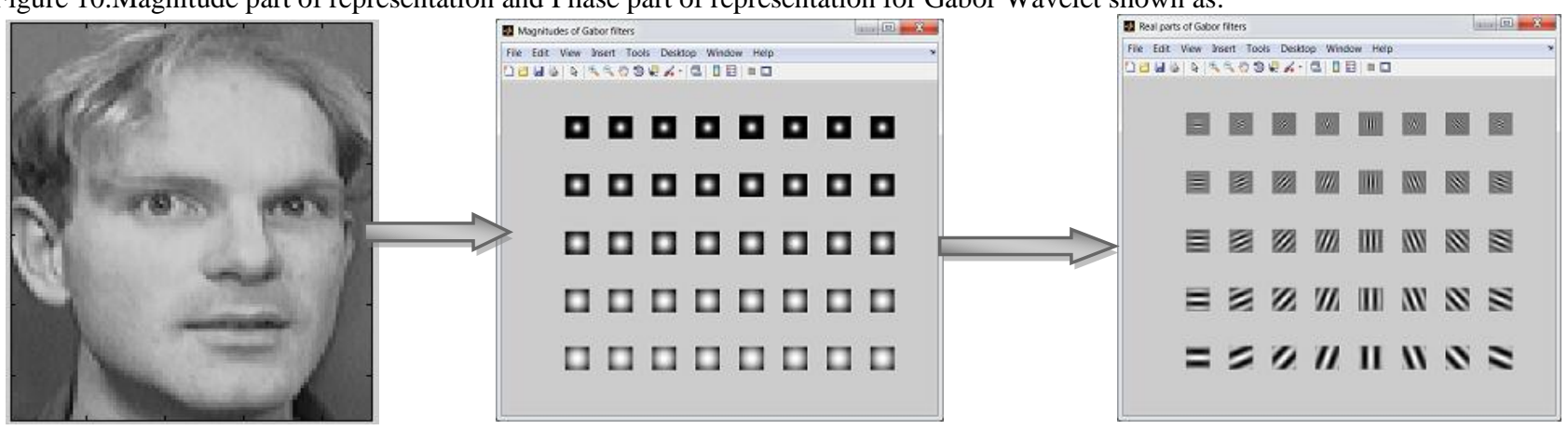

Fig 10 (a) Input image

Fig 10(b) Magnitude part of representation

Fig 10 (c) Phase part of representation

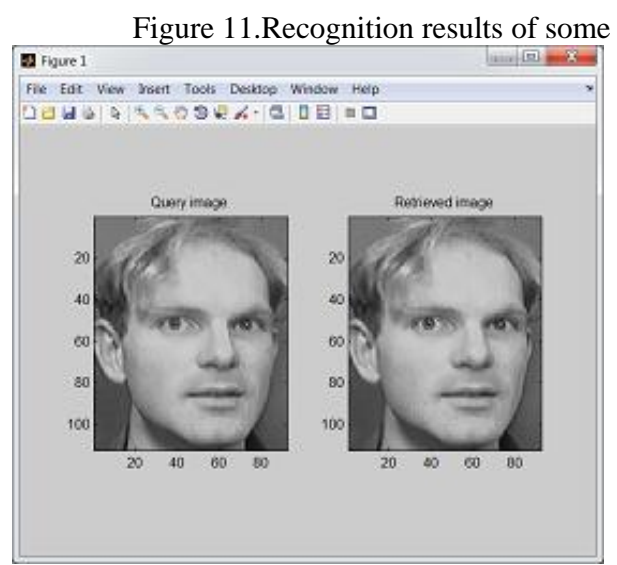

Figure 11(a)

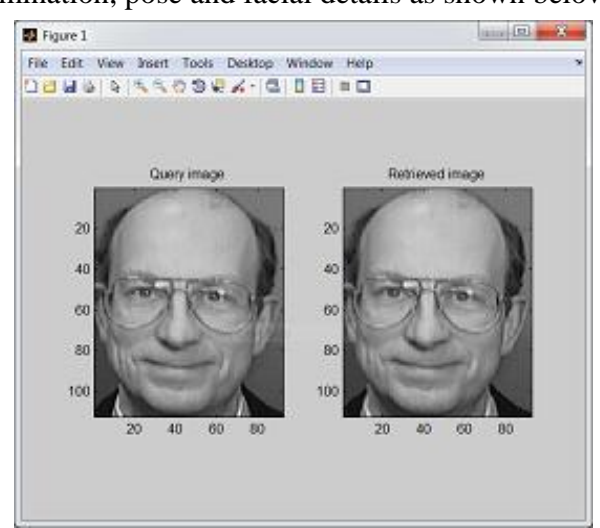

Figure 11(b) 


\section{CONCLUSION}

By implemented the face recognition and detection system by using combination of two approaches-PCA and Gabor Wavelet as, Gabor-Wavelet based PCA approach. This system worked better in different variations i.e. scale, orientation, expression and illumination of face for security purposes and successfully recognize and detect the human faces. To obtain an optimum result, threshold value can be used. We used this approach on ORL database. The system compares the vector characteristic of the test image with the different models contained in the database using the Euclidean distance as a classifier. The experimental results concluded that the proposed algorithm works better than traditional methods of face recognition and detection. Also, this approach is less time consuming, less memory occupying and faster technique and works well in constrained environment.

Although, the proposed work overcoming the major shortcomings as stated above, there are other peripheral shortcomings that influence the recognition rate includes: mental state (e.g., felt emotions or cogitation), verbal communication (that are communicated via illustrators responses of regulators and listeners),nonverbal communication(communicated via unfelt emotions and emblems etc.),physiological activities(such as via pain and tiredness or via manipulators). This indicates the need for more work to be done in the field of automated face recognition and detection systems with regard to the integration of other communication channels such as gesture and voice.

Finally, it can be suggested that if truly automatic face recognition and detection systems are to be feasible and efficient, current feature extraction methods have to be improved with regard to reliability, robustness in natural environments as well as independence of manual intervention during initialization and deployment.

\section{REFERENCES}

[1] Xiaoyang Tana, Songcan Chen, Zhi-Hua Zhou, Fuyan Zhang, " Face Recognition from a Single Image per Person: A survey", Chinese Academy of Sciences, Beijing 100080, Pattern Recognition 39 (2006).1725 1745 .

[2] R. Brunelli and T. Poggio, "Face recognition: features versus templates," IEEE Transactions on Pattern Analysis and Machine Intelligence, Vol.15, pp.10421052, 1993.

[3] M.A.Grudin, "On internal representations in face recognition systems," Pattern Recognition, Vol.33, pp.1161-1177, 2000

[4] B.Heisele, P. Ho, J. Wu, and T. Poggio,"Face recognition: component-based versus global approaches, "Computer Vision and Image Understanding, Vol.91, pp.6-21, 2003.

[5] A.M. Martinez, Recognizing imprecisely localized, partially occluded, and expression variant faces from a single sample per class, IEEE Trans. Pattern Anal. Mach. Intell. 25 (6) (2002) 748-763.

[6] A.M. Martinez, Recognizing expression variant faces from a single sample image per class, Proceedings of IEEE Computer Vision and Pattern Recognition (CVPR), 2003, pp.353-358.
[7] S. Lawrence, C.L. Giles, A. Tsoi, A. Back, Face recognition: a convolution neural-network approach, IEEE Trans. Neural Networks 8 (1)(1997) 98-113.

[8] X. Tan, S.C. Chen, Z.-H. Zhou, F. Zhang, Recognizing partially occluded, expression variant faces from single training image per person with SOM and soft kNN ensemble, IEEE Trans. Neural Networks 16 (4)(2005) 875-886.

[9] M. Zhou and H.Wei, "Face verification using Gabor wavelets and Adaboost".

[10] L. Shen and L. Bai,"Gabor feature based face recognition using kernel methods".

[11] P. Sankaran and K.V. Asari, "A multi-view approach on modular PCA for illumination and pose invariant face recognition".

[12] John Woodward, Christopher Horn, Julius Gatune, and Aryn Thomas,"Biometrics :A Look at Facial Recognition", Prepared for the Virginia State Crime Commission, 2003.

[13] RabiaJafri, and Hamid R. Arabnia,"A Survey of Face Recognition Techniques", Journal of Information Processing Systems, Vol.5, No.2, June 2009.

[14] Cahit Gurel, "Development of a face recognition system",Master dissertation, DEP: Mechatronics engineering, univ : Atýlým university,july 2011.

[15] B.S. Manjunath, R. Chellappa, C.V.D. Malsburg, A feature based approach to face recognition, in: Proceedings, IEEE Conference on Computer Vision and Pattern Recognition, vol. 1, 1992, pp. 373-378.

[16] Stelvio Cimato, Marco Gamassi, Vincenzo Piuri, Daniele Sana, Roberto Sassi, and Fabio Scotti," Personal identification and verification using multimodal biometric data",IEEE International Conference on Computational Intelligence for Homeland Security and Personal Safety Alexandria, VA, USA, 16-17 October 2006

[17] G. I. Davida, Y. Frankel, and B. J. Matt, "On enabling secure applications through off-line biometric," in Proceedings of the IEEE International Symposium on Security and Privacy, 1998. 1998, pp. 148-157, IEEE Press.

[18] K. J. Kantharia and G. I. Prajapati, "Facial Behavior Recognition Using So Computing Techniques: A Survey," 2015 Fifth International Conference on Advanced Computing \& CommunicationTechnologies, Haryana, 2015, pp.30-34.doi: 10.1109/ACCT.2015.132.

[19] Aye Pa Pa Mya and Myint MyintSein, "Tracking of the motion path of a person from video for the overlapping case," Instrumentation and Measurement Technology Conference, 2009.I2MTC '09. IEEE, Singapore, 2009, pp. 904-908.doi: 10.1109/IMTC.2009.5168579.

[20] Sakai, T., Nagao, M., and Fujibayashi, S. - Line extraction and pattern recognition in a photograph\|, Pattern Recognition, vol.1, pp. 233-248, 1969.

[21] Kelly, M, - Visual identification of people by computer\| Stanford AI Proj., Stanford, CA, Tech. Rep, 1970.

[22] W. W. W. Zou and P. C. Yuen, "Very Low Resolution Face Recognition Problem," in IEEE Transactions on 
Image Processing, vol. 21, no. 1, pp. 327-340, Jan. 2012. doi: 10.1109/TIP.2011.2162423.

[23] L. Fei-Fei, R. Fergus, and P. Perona, "Learning generative visual models from few training examples: an incremental Bayesian approach tested on101 object categories," IEEE. CVPR 2004, Workshop on Generative-Model BasedVision, 2004.

[24] Sample image database of about 500 images from Andrea Mosaic. [Online] Available: http://www.andreaplanet.com/andreamosaic/.

[25] A. K. Jain, R. Bolle, and S. Pankanti, "Biometrics: Personal Identification in Networked Security," A. K. Jain, R. Bolle, and S.Pankanti, Eds.: Kluwer Academic Publishers, 1999.

[26] J. Yang, X. Chen, and W. Kunz, "A PDA-based face recognition system," in Proceedings of sixth IEEE Workshop on Applications of Computer Vision. Orlando, Florida, 2002, pp.19-23.

[27] S. Gong, S. J. McKenna, and A. Psarrou., Dynamic Vision: From Images to Face Recognition: Imperial College Press (World Scientific Publishing Company), 2000.

[28] T. Jebara, "3D Pose Estimation andNormalization for Face Recognition," Center for Intelligent Machines, McGill University, Undergraduate Thesis May, 1996.

[29] P. J. Phillips, P. Grother, R. J. Micheals, D. M.Blackburn, E. Tabassi, and J. M. Bone, "Face Recognition Vendor Test (FRVT2002)," National Institute of Standards and Technology, Evaluation report IR6965, March, 2003.

[30] X. Q. Ding and C. Fang, "Discussions on some problems in face recognition," in Advances In Biometric Person Authentication, Proceedings, Vol. 3338, Lecture Notes In Computer Science: Springer Berlin / Heidelberg, 2004, pp.47-56.

[31] T. Kanade, "Picture Processing System by Computer Complex and Recognition of Human Faces," Kyoto University, Japan, PhD. Thesis 1973.

[32] L. Wiskott, J.-M.Fellous, N. Krüger, and C. von derMalsburg, "Face Recognition by Elastic Bunch Graph Matching," IEEETransactions on Pattern Analysis and Machine Intelligence, Vol.19, pp.775-779, 1997.

[33] L. Wiskott,R. Fellous, N. Kruger, C. von Malsburg, Face recognition by elastic bunch graph matching, IEEE Trans. Pattern Anal. Mach. Intell. 19 (7) (July 1997)775779.

[34] B.S. Manjunath, R. Chellappa, C.V.D. Malsburg, A feature based approach to face recognition, in: Proceedings, IEEE Conference on Computer Vision and Pattern Recognition, vol. 1, 1992, pp. 373-378.

[35] H.E. Komleh, V. Chandran, S. Sridharan, Robustness to expression variations in fractal-based face recognition, Proceedings ofISSPA-01, vol. 1, Kuala Lumpur, Malaysia, 13-16 August 2001, pp. 359-362.

[36] L. Sirovich and M. Kirby, "A Low-dimensional Procedure for the Characterization of Human Faces," Journal of the Optical Society of America: Optics, Image Science, and Vision, Vol.4, pp.519-524, 1987.
[37] R. A. Fisher, "The use of multiple measures in taxonomic problems," Annals of Eugenics, Vol.7, pp. 179-188, 1936.

[38] M. S. Bartlett, J. R. Movellan, and T. J. Sejnowski," Face recognition by independent component analysis," IEEE Transactions onNeural Networks, Vol.13, pp.14501464, 2002. [140] B. Draper, K. Baek, M. S.Bartlett

[39] P. Belhumer, J. Hespanha, D. Kriegman, "Eigenfaces vs. Fisher faces: Recognition using class specific linear projection", Proc. Of the Fourth European Conference on Computer Vision, vol.1,April 1996,Cambridge, UK, pp.45- 58

[40] M.S. Bartlett, J.R. Movellan, T.J. Sejnowski, "Face recognition by independent component analysis," IEEE Trans. on Neural Networks, vol.13, no.6, November 2002, pp.1450-1464.

[41] Liu CJ, Wechsler H (2002) Gabor feature based classification using the enhanced Fisher linear discriminant model for face recognition. IEEE Trans Image Process 11(4):467-476.

[42] Liu CJ, Wechsler H (2003) Independent component analysis of Gabor feature's for face recognition. IEEE Trans Neural Netw 14(4):919-928

[43] Liu CJ (2004) Gabor-based kernel PCA with fractional power polynomial models for face recognition. IEEE Trans PAMI 26(5):572-581.

[44] Shen L, Bai L, Fairhurst M (2006) Gabor wavelets and Generalized Discriminant Analysis for face identification and verification. Image Vis Compute (in press)

[45] J. Weng, N. Ahuja, and T. S. Huang, "Learning recognition and segmentation of 3-D objects from 3- D images," in Proceedings of the International Conference on Computer Vision (ICCV 93).Berlin, Germany, 1993 pp.121-128

[46] S. Lawrence, C. L. Giles, A. C. Tsoi, and A. D. Back, "Face Recognition: A Convolutional Neural Network Approach," IEEE Transactions on Neural Networks, Special Issue on Neural Networks and Pattern Recognition, pp.1-24, 1997

[47] A. Eleyan and H. Demirel, "Face RecognitionSystem Based on PCA and Feed forward Neural Networks," in Computational Intelligence and Bioinspired Systems, Vol.3512, Lecture Notes in Computer Science: Springer Berlin / Heidelberg, 2005, pp.935-942.

[48] G. C. Zhang, X. S. Huang, S. Z. Li, Y. S. Wang, and X. $\mathrm{H}$. Wu, "Boosting local binary pattern (LBP)- based face recognition," in Advances In Biometric Person Authentication, Proceedings, Vol.3338, Lecture Notes In Computer Science, 2004, pp.179-186.

[49] Y. Freund and R. E. Schapire, "A decision-theoretic generalization of on-line learning and an application to boosting," Journal of Computer and System Sciences, Vol.55, pp.119-139,1997

[50] F. S. Samaria and A. C. Harter, "Parameterization of a stochastic model for human face identification," in Proceedings of the 2ndIEEE Workshop on Applications of Computer Vision. Sarasota, FL, USA, 1994,pp.138142.

[51] Kruger V, Sommer G (2002) Wavelet networks for face processing. J Opt Soc Am A Opt Image Sci Vis 
19(6):1112- 1119 25. Jain AK, Farrokhnia F (1991) Unsupervised texture segmentation.

[52] Zhang $\mathrm{HH}$ et al (2005) Gabor wavelet associative memory for face recognition. IEEE Trans Neural Netw 16(1):275-278.

[53] A. Howell and H. Buxton, "Towards unconstrained face recognition from image sequences," in Proceedings of the Second IEEE International Conference on Automatic Face and Gesture Recognition, 1996, pp.224-229.

[54] T. E. de Campos, R. S. Feris, and R. M. Cesar Jr., "A Framework for Face Recognition from Video Sequences Using GWN and Eigen feature Selection," in Workshop on Artificial Intelligence and Computer Vision. Atibaia, Brazil, 2000

[55] S. Zhou and R. Chellappa, "Beyond a single still image: Face recognition from multiple still images and videos," in Face Processing: Advanced Modeling and Methods: Academic Press, 2005.

[56] A. Tibbalds, "Three Dimensional Human Face Acquisition for Recognition," Trinity College, University of Cambridge, Cambridge, Ph. D. Thesis March 1998.

[57] C. Hesher, A. Srivastava, and G. Erlebacher, "A novel technique for face recognition using range imaging," in Proceedings of the 7th IEEE International Symposium on Signal Processing and Its Applications, Vol.2, 2003, pp.201-204.

[58] Y. Wang, C. Chua, and Y. Ho, "Facial feature detection and face recognition from 2D and 3D images," Pattern Recognition Letters, Vol.23, pp.1191-1202, 2002.

[59] Y. Lee, H. Song, U. Yang, H. Shin, and K. Sohn, "Local feature based 3D face recognition," in Audio and VideoBased Biometric Person Authentication, Vol.3546, Lecture Notes in Computer Science: Springer Berlin / Heidelberg, 2005, pp.909-918.

[60] A. Ruifrok, A. Scheenstra, and R. C. Veltkamp, "A Survey of 3D Face Recognition Methods," in Audio and Video-based Biometric Person Authentication, Vol.3546, Lecture Notes in Computer Science: Springer Berlin / Heidelberg, 2005, pp.891-899.

[61] K. W. Bowyer, K. Chang, and P.J. Flynn, "A survey of approaches and challenges in $3 \mathrm{D}$ and multi-modal 3D+2Dface recognition," Computer Vision and Image Understanding, Vol.101, pp.1-15, 2006.

[62] M. Turk and A. Pentland, "Face Recognition Using Eigenfaces," in Proceedings of the IEEE Conference on Computer Vision and Pattern Recognition, 1991, pp.586591.

[63] M. Turk and A. Pentland, "Eigenfaces for Recognition," Journal Of Cognitive Neuroscience, Vol.3, pp.71-86, 1991.

[64] D. Socolinsky, L. Wolff, J. Neuheisel, and C. Eveland,"Illumination invariant face recognition using thermal infrared imagery," in IEEE Computer Society International Conference on Computer Vision and Pattern Recognition, Vol.1. Kauai, HI, USA, 2001, pp.527-534.
[65] R. Cutler, "Face recognition using infrared images and eigenfaces," University of Maryland at College Park, College Park, MD, USA, Technical report CSC 989, 1996.

[66] S. M. H. Anvar, W. Y. Yau and E. K.Teoh, "Multiview Face Detection and Registration Requiring Minimal Manual Intervention," in IEEE Transactions on Pattern Analysis and Machine Intelligence, vol. 35, no. 10, pp. 2484-2497, Oct. 2013. doi:10.1109/TPAMI.2013.37

[67] Bo Wu, Haizhou Ai, Chang Huang and Shihong Lao, "Fast rotation invariant multi-view face detection based on real Adaboost," Automatic Face and Gesture Recognition, 2004.Proceedings. Sixth IEEE International Conference on, 2004, pp. 79-84.doi: 10.1109/AFGR.2004.1301512

[68] M. A. Tinati, E. Namjoo and M. B. A. Haghighat, "Evaluating the effect of the eigen values on BDF classifier in face detection," Application of Information and Communication Technologies (AICT), 2011 5th International Conference on, Baku, 2011, pp. 1-5.doi: 10.1109/ICAICT.2011.6111008

[69] Chengjun Liu, "A Bayesian discriminating features method for face detection, " in IEEE Transactions on Pattern Analysis and Machine Intelligence, vol. 25, no. 6, pp. 725-740, June 2003.doi: 10.1109/TPAMI.2003.1201822

[70] S. Z. Li and Zhenqiu Zhang, "Float Boost learning and statistical face detection ," in IEEE Transactions on Pattern Analysis and Machine Intelligence, vol. 26, no. 9, pp. 1112-1123, Sept. 2004.doi: 10.1109/TPAMI.2004.68

[71] Vladimir VN, "The Nature of Statistical Learning Theory. Springer", Berlin Heidelberg New York, 1995.

[72] Cascade Classification - OpenCV 2.4.13.3 documentation http://docs.opencv.org/modules/objdetect/ doc/cascade-classification.html

[73] DATABASES http://www.face-rec.org/databases

[74] L. Sirovich and M Kirby, "A low dimensional procedure for the characterization of human faces", JOSA A 4, no. 3 (1987): 519-524.

[75] R. Gottumukkal, K.V. Asari, "An improved face recognition technique based on modular PCA approach," Pattern recog.Letters,vol.25, 2004, pp.429-436.

[76] P.N. Belhumeur and J.P. Hespanha and D.J. Kriegman,"Eigenfaces vs. Fisherfaces: Recognition using class specific linear projection", PAMI Special Issue on Face Recognition, vol. 19, no. 7, July1997, pp. 711-720.

[77] Z.L. Zheng, F.Yang, W.A. Tan, J. Jia, J. Yang, "Gabor feature-based face recognition using supervised locality preserving projection," Signal Processing, vol. 87, 2007, pp. 2473-2483.

[78] X. Pan, Q.Q. Ruan, "Palmprint recognition using Gabor feature based (2D) 2PCA," Neurocomputing, vol.71 (1315), 2008, pp. 3032- 3036

[79] L.L. Shen, L.Bai, Michael Fairhurst, "Gabor wavelets and general discriminated analysis for face identification and verification, "Images and Vision Computing vol. 25(5), 2007, pp.553-563. 